\title{
Existence of Sign-Changing Solutions to Equations Involving the One-Dimensional $p$-Laplacian
}

\author{
Ruyun Ma and Lingfang Jiang \\ Department of Mathematics, Northwest Normal University, Lanzhou 730070, China \\ Correspondence should be addressed to Ruyun Ma; ruyun_ma@126.com
}

Received 23 March 2014; Accepted 21 July 2014; Published 17 August 2014

Academic Editor: Mehmet Sezer

Copyright (C) 2014 R. Ma and L. Jiang. This is an open access article distributed under the Creative Commons Attribution License, which permits unrestricted use, distribution, and reproduction in any medium, provided the original work is properly cited.

We consider the equations involving the one-dimensional $p$-Laplacian $(P):\left(\left|u^{\prime}(t)\right|^{p-2} u^{\prime}(t)\right)^{\prime}+\lambda f(u(t))=0,0<t<1$, and $u(0)=u(1)=0$, where $p>1, \lambda>0, f \in C^{1}(\mathbb{R} ; \mathbb{R}), f(s) s>0$, and $s \neq 0$. We show the existence of sign-changing solutions under the assumptions $f_{\infty}=\lim _{|s| \rightarrow \infty}\left(f(s) / s^{p-1}\right)=+\infty$ and $f_{0}=\lim _{|s| \rightarrow 0}\left(f(s) / s^{p-1}\right) \in[0, \infty]$. We also show that $(P)$ has exactly one solution having specified nodal properties for $\lambda \in\left(0, \lambda^{*}\right)$ for some $\lambda^{*} \in(0, \infty)$. Our main results are based on quadrature method.

\section{Introduction and Main Results}

Existence and multiplicity of positive solutions of nonlinear second order boundary value problem

$$
\begin{gathered}
u^{\prime \prime}(t)+\lambda f(u(t))=0, \quad 0<t<1, \\
u(0)=u(1)=0
\end{gathered}
$$

and its generalized forms have been extensively studied via the fixed point theorem in cones, bifurcation theory, quadrature method, and fixed index theorem in the past four decades; see Erbe and Wang [1], Henderson and Wang [2], Laetsch [3], Fink et al. [4], Ma and Thompson [5, 6], and the references therein.

Existence and multiplicity of positive solutions of the corresponding one-dimensional $p$-Laplacian

$$
\begin{gathered}
\left(\left|u^{\prime}(t)\right|^{p-2} u^{\prime}(t)\right)^{\prime}+\lambda a(t) f(u(t))=0, \quad 0<t<1, \\
u(0)=u(1)=0
\end{gathered}
$$

have also been studied by several authors; see Lee and Sim [7], Wang [8], Kong and Wang [9], Aranda and Godoy [10], Bouguima and Lakmeche [11], and de Coster [12] for references along this line.
Recently, Lee and Sim [7] consider the existence and multiplicity of positive solutions of (2), (3) under the assumptions

$$
f_{\infty}=\infty, \quad f_{0} \in(0, \infty) .
$$

They proved the following.

Theorem A (see [7, Theorem 3.14]). Assume (4) hold. Then, there exist $\lambda_{*} \geq \lambda^{*}>0$ such that (2), (3) have at least one positive solution for $\lambda<\lambda^{*}$ and no positive solution for $\lambda>\lambda_{*}$.

Of course, natural question is as follows. What would happen if we allow that $f_{0} \in\{0, \infty\}$ ?

It is the purpose of this paper to study sign-changing solutions of (2), (3) under the assumptions $a(t) \equiv 1$ and

$$
f_{\infty}=\infty, \quad f_{0}=0,
$$

or

$$
f_{\infty}=\infty, \quad f_{0}=\infty .
$$

The main tool is the quadrature method.

We will make the following assumptions:

(H0) $f(s) s>0$ for $s \neq 0$;

(H1) $\lim _{|s| \rightarrow \infty}\left(f(s) / s^{p-1}\right)=+\infty$;

(H2) $\lim _{s \rightarrow+\infty}\left(((p-1) / p) f(s)-(s / p) f^{\prime}(s)\right)<0$ and $\lim _{s \rightarrow-\infty}\left(((p-1) / p) f(s)-(s / p) f^{\prime}(s)\right)>0$. 
Let $\lambda_{k}:=k^{p} \pi_{p}^{p}, k \in \mathbb{N}$, where $\pi_{p}:=2 \int_{0}^{(p-1)^{1 / p}} 1 /(1-$ $\left.\left(s^{p} /(p-1)\right)\right)^{1 / p} d s$. The main results of this paper are the following.

Theorem 1. Let (HO), (H1), and (H2) hold. Assume that $f$ satisfies

$$
f_{0}=\lim _{|s| \rightarrow 0} \frac{f(s)}{s^{p-1}} \in(0, \infty) .
$$

Then, for $k \in \mathbb{N}$, (2), (3) have two solutions $u_{k}^{+}$and $u_{k}^{-}$for each $\lambda \in\left(0, \lambda_{k} / f_{0}\right): u_{k}^{+}$has $k-1$ zeros in $(0,1)$ and is positive near 0 , and $u_{k}^{-}$has $k-1$ zeros in $(0,1)$ and is negative near 0 . Moreover, there exists a constant $\lambda^{*} \in\left(0, \lambda_{k} / f_{0}\right)$, such that for each $\lambda \epsilon$ $\left(0, \lambda^{*}\right)$ the above solution is unique.

Theorem 2. Let (HO), (H1), and (H2) hold. Assume that $f_{0}=$ 0 . Then, for $k \in \mathbb{N}$, (2), (3) have two solutions $u_{k}^{+}$and $u_{k}^{-}$for each $\lambda \in(0, \infty)$ : $u_{k}^{+}$has $k-1$ zeros in $(0,1)$ and is positive near 0 , and $u_{k}^{-}$has $k-1$ zeros in $(0,1)$ and is negative near 0 . Further, there exists a constant $\lambda^{*} \in(0, \infty)$ independent of $k$, such that for each $\lambda \in\left(0, \lambda^{*}\right)$ the above solution is unique.

Theorem 3. Let (HO) and (H1) hold. Assume that $f_{0}=\infty$. Then, for $k \in \mathbb{N}$, there exists a constant $\lambda_{*}$ small independent of $k$, such that for each $\lambda \in\left(0, \lambda_{*}\right)(2)$, (3) have two solutions $u_{k}^{+}$ and $v_{k}^{+}: u_{k}^{+}$and $v_{k}^{+}$have $k-1$ zeros in $(0,1)$ and are positive near 0 ; and problems (2), (3) have two solutions $u_{k}^{-}$and $v_{k}^{-}$, where $u_{k}^{-}$and $v_{k}^{-}$have $k-1$ zeros in $(0,1)$ and are negative near 0.

Remark 4. For $p=2$, the existence of positive and signchanging solutions has been extensively studied by many authors [1-6], but they did not give any information about the uniqueness of nodal solutions.

Remark 5. It is worth noticing that Lee and Sim [7] studied the nonautonomous cases (2), (3) and obtained the existence of positive solutions with $f_{\infty}=\infty, f_{0} \in(0, \infty)$. They gave no information about the sign-changing solutions. In Theorem 1 , we show the existence of solutions having specified nodal properties.

Remark 6. Very little is known in the available literature even in the special case $p=2$. We establish uniqueness results in this paper; see Theorems 1 and 2 .

Remark 7. Let us consider the problem

$$
\begin{gathered}
\left(\left|u^{\prime}(t)\right|^{8 / 3-2} u^{\prime}(t)\right)^{\prime}+\lambda g(u(t))=0, \quad 0<t<1, \\
u(0)=u(1)=0,
\end{gathered}
$$

where $g(s)=s^{11 / 3}+s^{5 / 3}$. Obviously, $g$ satisfies (H0) and (H1). Since

$$
\frac{8 / 3-1}{8 / 3} g(s)-\frac{s}{8 / 3} g^{\prime}(s)=-\frac{3}{4} s^{11 / 3},
$$

it is easy to see that (H2) is fulfilled. Thus, Theorem 1 implies that, for $k \in \mathbb{N}$, (6) have two solutions $u_{k}^{+}$and $u_{k}^{-}$for each $\lambda \in\left(0, \lambda_{k} / f_{0}\right): u_{k}^{+}$has $k-1$ zeros in $(0,1)$ and is positive near 0 , and $u_{k}^{-}$has $k-1$ zeros in $(0,1)$ and is negative near 0 . Moreover, there exists a constant $\lambda^{*} \in\left(0, \lambda_{k} / f_{0}\right)$, such that for each $\lambda \in\left(0, \lambda^{*}\right)$ the above solution is unique.

For other results dealing with $p$-Laplacian operators and the bifurcation behavior of solutions, see [13-24] and the references therein.

The rest of the paper is arranged as follows. In Section 2, we state and prove some preliminary results. Finally, in Section 3, we give the proofs of Theorems 1, 2, and 3.

\section{Quadrature Method and Preliminaries}

Let $f \in C^{1}(\mathbb{R} ; \mathbb{R}), f(s) s>0$ for $s \neq 0$ and $F(s)=\int_{0}^{s} f(t) d t$.

Lemma 8. If $u$ is any solution of (2), (3) and $x_{0} \in(0,1)$ is such that $u^{\prime}\left(x_{0}\right)=0$, then $u\left(x_{0}-t\right)=u\left(x_{0}+t\right), t \in\left[0, \min \left\{x_{0}, 1-\right.\right.$ $\left.x_{0}\right\}$.

Proof. Since $f$ is autonomous, both $u\left(x_{0}-t\right)$ and $u\left(x_{0}+t\right)$ satisfy the initial value problem

$$
\begin{array}{r}
\left(\left|w^{\prime}(t)\right|^{p-2} w^{\prime}(t)\right)^{\prime}+\lambda f(w(t))=0, \\
t \in\left[0, \min \left\{x_{0}, 1-x_{0}\right\}\right], \\
w(0)=u\left(x_{0}\right), \quad w^{\prime}(0)=0 .
\end{array}
$$

By Reichel and Walter [14, Theorem 2] and [14, (iii) and (v) in the case $(\beta)$ of Theorem 4$]$, (8) has a unique solution defined on $t \in\left(0, \min \left\{x_{0}, 1-x_{0}\right\}\right)$. Therefore, $u\left(x_{0}-t\right)=u\left(x_{0}+t\right)$.

Now, we divide the discussion into two cases.

Case $1(k=2 n+1$.). In this case, we attempt to find a solution of (2), (3) with $2 n$ zeros in $(0,1)$ and $u^{\prime}(0)<0$ and a solution of (2), (3) with $2 n$ zeros in $(0,1)$ and $u^{\prime}(0)>0$.

Obviously, if $u$ is a sign-changing solution with $2 n$ zeros in $(0,1)$ and $u^{\prime}(0)<0$, then, thanks to Lemma 8 and the fact that (2) is autonomous, we only need to study $u$ on the intervals $\left[x_{0}, 2 x_{0}\right]$ and $\left[2 x_{0}, 1 / 2 n+((n-1) / n) x_{0}\right]$.

Multiplying (2) throughout by $u^{\prime}(t)$, we obtain

$$
\left(\left|u^{\prime}\right|^{p-2} u^{\prime}(t)\right)^{\prime} u^{\prime}(t)+\lambda f(u) u^{\prime}(t)=0,
$$

and integrating we have

$$
\left|u^{\prime}\right|^{p}=-\lambda \frac{p}{p-1} F(u(t))+\frac{p}{p-1} c .
$$

If $h=\sup _{t \in[0,1]} u(t)$ and $q=\inf _{t \in[0,1]} u(t)$, then $u\left(x_{0}\right)=q$ and $u\left(1 / 2 n+((n-1) / n) x_{0}\right)=h$. Substituting $t=x_{0}$ and $t=$ $1 / 2 n+((n-1) / n) x_{0}$ in $(10)$, we get $c=\lambda F(q)$ and $c=\lambda F(h)$. Hence, $q=q(h)$ is such that

$$
F(q)=F(h) .
$$


Thus, we have

$$
\begin{gathered}
u^{\prime}(t)=\left(\frac{p}{p-1} \lambda\right)^{1 / p}(F(h)-F(u))^{1 / p} ; \\
t \in\left(2 x_{0}, \frac{1}{2 n}+\frac{n-1}{n} x_{0}\right), \\
u^{\prime}(t)=\left(\frac{p}{p-1} \lambda\right)^{1 / p}(F(q)-F(u))^{1 / p} ; \quad t \in\left(x_{0}, 2 x_{0}\right) .
\end{gathered}
$$

Integrating (12) and (13) on $\left(2 x_{0}, 1 / 2 n+((n-1) / n) x_{0}\right)$ and $\left(x_{0}, 2 x_{0}\right)$, respectively, we obtain

$$
\begin{aligned}
& \int_{0}^{u(t)} \frac{d u}{(F(h)-F(u))^{1 / p}}=\left(\frac{p}{p-1} \lambda\right)^{1 / p}\left(t-2 x_{0}\right), \\
& \int_{u(t)}^{0} \frac{d u}{(F(q)-F(u))^{1 / p}}=\left(\frac{p}{p-1} \lambda\right)^{1 / p}\left(2 x_{0}-t\right) .
\end{aligned}
$$

Hence, substituting $t=1 / 2 n+((n-1) / n) x_{0}$ in (14) and $t=x_{0}$ in (15), we have

$$
\begin{gathered}
\int_{0}^{h} \frac{d u}{(F(h)-F(u))^{1 / p}}=\left(\frac{p}{p-1} \lambda\right)^{1 / p}\left(\frac{1}{2 n}-\frac{n+1}{n} x_{0}\right), \\
\int_{q}^{0} \frac{d u}{(F(q)-F(u))^{1 / p}}=\left(\frac{p}{p-1} \lambda\right)^{1 / p} x_{0} .
\end{gathered}
$$

Multiplying (17) by $(n+1) / n$ and adding to (16), we can see that $\lambda$ and $h$ satisfy

$$
\begin{aligned}
(\lambda)^{1 / p}=2\left(\frac{p-1}{p}\right)^{1 / p} & \left\{n \int_{0}^{h} \frac{d u}{(F(h)-F(u))^{1 / p}}\right. \\
& \left.+(n+1) \int_{q}^{0} \frac{d u}{(F(q)-F(u))^{1 / p}}\right\} \\
& :=G_{2 n}(h) .
\end{aligned}
$$

In fact, the following result holds.

Lemma 9. Given $\lambda>0$, if there exists $h \in(0, \infty)$ such that $G_{2 n}(h)=(\lambda)^{1 / p}$, then (2), (3) have a sign-changing solution with $2 n$ interior zeros satisfying $\|u\|=\sup _{t \in(0,1)} u(t)=h$. Further, $G_{2 n}(h)$ is a continuous function in $(0, \infty)$ and it is also differentiable with the derivative given by

$$
\begin{aligned}
\frac{d G_{2 n}(h)}{d h}= & 2\left(\frac{p-1}{p}\right)^{1 / p} \\
\times & \left\{n \int_{0}^{1} \frac{H(h)-H(h v)}{(F(h)-F(h v))^{(p+1) / p}} d v\right. \\
& \left.-(n+1) \frac{d q}{d h} \int_{0}^{1} \frac{H(q)-H(q v)}{(F(q)-F(q v))^{(p+1) / p}} d v\right\},
\end{aligned}
$$

where $H(s)=F(s)-(s / p) f(s)$.
The proof of the above theorem follows by carefully extending the arguments used in [15, Theorem 2.2] for second order differential equation to the case of one-dimensional $p$ Laplacian.

Using the same argument, with obvious changes, we may deduce the following.

If $u$ is a sign-changing solution with $2 n$ zeros in $(0,1)$ and $u^{\prime}(0)>0$, the corresponding $G 1_{2 n}(h)$ is

$$
\begin{aligned}
G 1_{2 n}(h)= & 2\left(\frac{p-1}{p}\right)^{1 / p} \\
& \times\left\{(n+1) \int_{0}^{h} \frac{d u}{(F(h)-F(u))^{1 / p}}\right. \\
& \left.+n \int_{q}^{0} \frac{d u}{(F(q)-F(u))^{1 / p}}\right\} .
\end{aligned}
$$

Case $2(k=2 n)$. In this case, if $u$ is a sign-changing solution with $2 n-1$ zeros in $(0,1)$ and $u^{\prime}(0)>0$, the corresponding $G_{2 n-1}(h)$ is

$$
\begin{aligned}
& G_{2 n-1}(h)=(\lambda)^{1 / p} \\
&=2\left(\frac{p-1}{p}\right)^{1 / p} \times\left\{n \int_{0}^{h} \frac{d u}{(F(h)-F(u))^{1 / p}}\right. \\
&\left.+n \int_{q}^{0} \frac{d u}{(F(q)-F(u))^{1 / p}}\right\} .
\end{aligned}
$$

Similarly, we may get the same function $G_{2 n-1}(h)$ as above when $u$ is a sign-changing solution with $(2 n-1)$ zeros in $(0,1)$ with $u^{\prime}(0)<0$.

\section{The Proofs of the Main Results}

Proof of Theorem 1. First, we consider $k=2 n+1$.

It follows from the quadrature method that a solution with $2 n$ zeros in $(0,1)$ exists if for $\lambda>0$ there exists $h \in(0, \infty)$ such that $(\lambda)^{1 / p}=G_{2 n}(h)$. To prove this, we will show that $\left(0,(2 n+1) \pi_{p} / f_{0}^{1 / p}\right) \subset \operatorname{Range}\left(G_{2 n}(h)\right)$. We achieve this by proving
(A) $\lim _{h \rightarrow+\infty} G_{2 n}(h)=0$,
(B) $\lim _{h \rightarrow 0} G_{2 n}(h)=(2 n+1) \pi_{p} / f_{0}^{1 / p}$.

Proof of (A). Recall that

$$
\begin{aligned}
G_{2 n}(h)= & 2\left(\frac{p-1}{p}\right)^{1 / p} \\
& \times\left\{n \int_{0}^{h} \frac{d u}{(F(h)-F(u))^{1 / p}}\right. \\
& \left.+(n+1) \int_{q}^{0} \frac{d u}{(F(q)-F(u))^{1 / p}}\right\} .
\end{aligned}
$$


First let us consider

$$
\begin{aligned}
& 2\left(\frac{p-1}{p}\right)^{1 / p} n \int_{0}^{h} \frac{d u}{(F(h)-F(u))^{1 / p}} \\
& \quad=2\left(\frac{p-1}{p}\right)^{1 / p} n \int_{0}^{h} \frac{d u}{\left(\int_{u}^{h} f(t) d t\right)^{1 / p}} .
\end{aligned}
$$

To this end, we have from (H1) that, for any $k \in \mathbb{N}$, there exists $R_{k} \in(0, \infty)$, such that

$$
f(s) \geq k^{p} s^{p-1}, \quad s \geq R_{k} .
$$

If $h>R_{k}$, it follows from (23) and (24) that we have that

$$
\begin{aligned}
& 2\left(\frac{p-1}{p}\right)^{1 / p} \int_{0}^{R_{k}} \frac{d s}{(F(h)-F(s))^{1 / p}} \\
& +2\left(\frac{p-1}{p}\right)^{1 / p} n \int_{R_{k}}^{h} \frac{d s}{(F(h)-F(s))^{1 / p}} \\
& =2\left(\frac{p-1}{p}\right)^{1 / p} n \int_{0}^{R_{k}} \frac{d s}{\left(\int_{s}^{h} f(w) d w\right)^{1 / p}} \\
& +2\left(\frac{p-1}{p}\right)^{1 / p} n \int_{R_{k}}^{h} \frac{d s}{\left(\int_{s}^{h} f(w) d w\right)^{1 / p}} \\
& \leq 2\left(\frac{p-1}{p}\right)^{1 / p} n \int_{0}^{R_{k}}\left(\frac{k^{p}}{p}\left(h^{p}-R_{k}^{p}\right)\right)^{-1 / p} d s \\
& +2\left(\frac{p-1}{p}\right)^{1 / p} n \int_{R_{k}}^{h}\left(\frac{k^{p}}{p}\left(h^{p}-s^{p}\right)\right)^{-1 / p} d s \\
& =2(p-1)^{1 / p} n \frac{1}{k} \\
& \times\left\{R_{k}\left[\left(h^{p}-R_{k}^{p}\right)\right]^{-1 / p}+\int_{R_{k} / h}^{1} \frac{1}{\left(1-v^{p}\right)^{1 / p}} d v\right\} \\
& =n \frac{1}{k}\left\{2(p-1)^{1 / p} R_{k}\left[\left(h^{p}-R_{k}^{p}\right)\right]^{-1 / p}\right. \\
& \left.+2 \int_{R_{k}(p-1)^{1 / p} / h}^{(p-1)^{1 / p}} \frac{1}{\left(1-\left(v^{p} /(p-1)\right)\right)^{1 / p}} d v\right\} \\
& \longrightarrow \frac{n C}{k} \text { as } h \longrightarrow \infty \text {, }
\end{aligned}
$$

where

$$
\begin{aligned}
C:=\lim _{h \rightarrow \infty}\left\{2(p-1)^{1 / p} R_{k}\left[\left(h^{p}-R_{k}^{p}\right)\right]^{-1 / p}\right. \\
\left.+2 \int_{R_{k}(p-1)^{1 / p} / h}^{(p-1)^{1 / p}} \frac{1}{\left(1-\left(v^{p} /(p-1)\right)\right)^{1 / p}} d v\right\}
\end{aligned}
$$

It follows from the fact that $k$ is sufficiently large and (25) that

$$
2\left(\frac{p-1}{p}\right)^{1 / p} n \int_{0}^{h} \frac{d u}{(F(h)-F(u))^{1 / p}}=0
$$

Next, we know that $q \rightarrow-\infty$ as $h \rightarrow+\infty(F(q)=F(h))$. We consider

$$
\begin{aligned}
& 2\left(\frac{p-1}{p}\right)^{1 / p}(n+1) \int_{q}^{0} \frac{d u}{(F(q)-F(u))^{1 / p}} \\
& \quad=2\left(\frac{p-1}{p}\right)^{1 / p}(n+1) \int_{q}^{0} \frac{d u}{\left(\int_{u}^{q} f(t) d t\right)^{1 / p}}
\end{aligned}
$$

To this end, we have from (H1) that, for any $k \in \mathbb{N}$, there exists $R_{k} \in(0, \infty)$, such that

$$
-f(s) \geq k^{p}|s|^{p-1}, \quad s \leq-R_{k}
$$

If $q<-R_{k}$, it follows from (28) and (29) that we have that

$$
\begin{aligned}
& 2\left(\frac{p-1}{p}\right)^{1 / p}(n+1) \int_{q}^{-R_{k}} \frac{d s}{(F(q)-F(s))^{1 / p}} \\
& +2\left(\frac{p-1}{p}\right)^{1 / p}(n+1) \int_{-R_{k}}^{0} \frac{d s}{(F(q)-F(s))^{1 / p}} \\
& =2\left(\frac{p-1}{p}\right)^{1 / p}(n+1) \int_{q}^{-R_{k}} \frac{d s}{\left(\int_{s}^{q} f(w) d w\right)^{1 / p}} \\
& +2\left(\frac{p-1}{p}\right)^{1 / p}(n+1) \int_{-R_{k}}^{0} \frac{d s}{\left(\int_{s}^{q} f(w) d w\right)^{1 / p}} \\
& \leq 2\left(\frac{p-1}{p}\right)^{1 / p}(n+1) \int_{-R_{k}}^{0}\left(\frac{k^{p}}{p}\left(|q|^{p}-R_{k}^{p}\right)\right)^{-1 / p} d s \\
& +2\left(\frac{p-1}{p}\right)^{1 / p}(n+1) \int_{q}^{-R_{k}}\left(\frac{k^{p}}{p}\left(|q|^{p}-|s|^{p}\right)\right)^{-1 / p} d s \\
& =2(p-1)^{1 / p} \frac{(n+1)}{k}\left\{R_{k}\left[\left(|q|^{p}-R_{k}^{p}\right)\right]^{-1 / p}\right. \\
& \left.+\int_{-R_{k} / q}^{1} \frac{1}{\left(1-v^{p}\right)^{1 / p}} d v\right\} \\
& =\frac{(n+1)}{k}\left\{2(p-1)^{1 / p} R_{k}\left[\left(|q|^{p}-R_{k}^{p}\right)\right]^{-1 / p}\right.
\end{aligned}
$$




$$
\begin{gathered}
\left.+2 \int_{-R_{k}(p-1)^{1 / p} / q}^{(p-1)^{1 / p}} \frac{1}{\left(1-\left(v^{p} /(p-1)\right)\right)^{1 / p}} d v\right\} \\
\longrightarrow \frac{(n+1) C}{k} \text { as } q \longrightarrow-\infty
\end{gathered}
$$

where

$$
\begin{aligned}
C:=\lim _{q \rightarrow-\infty} & \left\{2(p-1)^{1 / p} R_{k}\left[\left(|q|^{p}-R_{k}^{p}\right)\right]^{-1 / p}\right. \\
& \left.+2 \int_{-R_{k}(p-1)^{1 / p} / q}^{(p-1)^{1 / p}} \frac{1}{\left(1-\left(v^{p} /(p-1)\right)\right)^{1 / p}} d v\right\} \\
= & \pi_{p} .
\end{aligned}
$$

It follows from the fact that $k$ is sufficiently large and (30) that

$$
\sqrt{2}(n+1) \int_{q}^{0} \frac{d u}{(F(q)-F(u))^{1 / 2}}=0 .
$$

Therefore, from (27) and (32), we have that $\lim _{h \rightarrow \infty} G_{2 n}(h)=$ 0 .

Proof of (B). Recall that

$$
\begin{aligned}
G_{2 n}(h)=2\left(\frac{p-1}{p}\right)^{1 / p} & \left\{n \int_{0}^{h} \frac{d u}{(F(h)-F(u))^{1 / p}}\right. \\
& \left.+(n+1) \int_{q}^{0} \frac{d u}{(F(q)-F(u))^{1 / p}}\right\} .
\end{aligned}
$$

First, let us consider

$$
\begin{aligned}
& 2\left(\frac{p-1}{p}\right)^{1 / p} n \int_{0}^{h} \frac{d u}{(F(h)-F(u))^{1 / p}} \\
& \quad=2\left(\frac{p-1}{p}\right)^{1 / p} n \int_{0}^{h} \frac{d u}{\left(\int_{u}^{h} f(t) d t\right)^{1 / p}} .
\end{aligned}
$$

Since $f_{0} \in(0, \infty)$, then, for any $\varepsilon \in\left(0, f_{0} / 2\right)$, there exists $\delta \in(0, \infty)$ such that

$$
f_{0}-\varepsilon \leq \frac{f(s)}{s^{p-1}} \leq f_{0}+\varepsilon, \quad 0<s<\delta .
$$

Thus, if $0<h<\delta$, the second part of (35) implies that

$$
\begin{aligned}
& 2 n\left(\frac{p-1}{p}\right)^{1 / p} \int_{0}^{h} \frac{1}{\left(\int_{u}^{h} f(v) d v\right)^{1 / p}} d u \\
& \quad \geq 2 n\left(\frac{p-1}{p}\right)^{1 / p}\left(\frac{p}{f_{0}+\varepsilon}\right)^{1 / p} \int_{0}^{h} \frac{1}{\left(h^{p}-u^{p}\right)^{1 / p}} d u
\end{aligned}
$$

$$
\begin{aligned}
& =2 n\left(\frac{p-1}{f_{0}+\varepsilon}\right)^{1 / p} \int_{0}^{h} \frac{1}{\left(h^{p}-u^{p}\right)^{1 / p}} d u \\
& =2 n\left(\frac{p-1}{f_{0}+\varepsilon}\right)^{1 / p} \frac{1}{(p-1)^{1 / p}}
\end{aligned}
$$$$
\times \int_{0}^{(p-1)^{1 / p}} \frac{1}{\left(1-\left(u^{p} /(p-1)\right)\right)^{1 / p}} d u
$$$$
=\frac{2 n}{\left(f_{0}+\varepsilon\right)^{1 / p}} \int_{0}^{(p-1)^{1 / p}} \frac{1}{\left(1-\left(u^{p} /(p-1)\right)\right)^{1 / p}} d u
$$$$
=\frac{n \pi_{p}}{\left(f_{0}+\varepsilon\right)^{1 / p}} \text {. }
$$

Similarly, from the first part of (35), we have that

$$
\begin{aligned}
2 n & \left(\frac{p-1}{p}\right)^{1 / p} \int_{0}^{h} \frac{1}{\left(\int_{u}^{h} f(v) d v\right)^{1 / p}} d u \\
\leq & 2 n\left(\frac{p-1}{p}\right)^{1 / p}\left(\frac{p}{f_{0}-\varepsilon}\right)^{1 / p} \int_{0}^{h} \frac{1}{\left(h^{p}-u^{p}\right)^{1 / p}} d u \\
= & 2 n\left(\frac{p-1}{f_{0}-\varepsilon}\right)^{1 / p} \int_{0}^{h} \frac{1}{\left(h^{p}-u^{p}\right)^{1 / p}} d u \\
= & 2 n\left(\frac{p-1}{f_{0}-\varepsilon}\right)^{1 / p} \frac{1}{(p-1)^{1 / p}} \\
& \times \int_{0}^{(p-1)^{1 / p}} \frac{1}{\left(1-\left(u^{p} /(p-1)\right)\right)^{1 / p}} d u \\
= & \frac{2 n}{\left(f_{0}-\varepsilon\right)^{1 / p}} \int_{0}^{(p-1)^{1 / p}} \frac{1}{\left(1-\left(u^{p} /(p-1)\right)\right)^{1 / p}} d u \\
= & \frac{n \pi_{p}}{\left(f_{0}-\varepsilon\right)^{1 / p}} .
\end{aligned}
$$

It follows from (36), (37) and the fact $\varepsilon$ is arbitrary that

$$
\lim _{h \rightarrow 0} 2\left(\frac{p-1}{p}\right)^{1 / p} n \int_{0}^{h} \frac{d u}{(F(h)-F(u))^{1 / p}}=\frac{n \pi_{p}}{f_{0}^{1 / p}} .
$$

In fact, $q \rightarrow 0$ as $h \rightarrow 0(F(h)=F(q))$; we consider

$$
2\left(\frac{p-1}{p}\right)^{1 / p}(n+1) \int_{q}^{0} \frac{d u}{(F(q)-F(u))^{1 / p}} .
$$

From $f_{0} \in(0, \infty)$, then, for any $\varepsilon \in\left(0, f_{0} / 2\right)$, there exists $\delta \in(0, \infty)$ such that

$$
\left(f_{0}-\varepsilon\right)|s|^{p-1} \leq-f(s) \leq\left(f_{0}+\varepsilon\right)|s|^{p-1}, \quad-\delta<s<0 .
$$


Thus, if $-\delta<q<0$, the second part of (40) implies that

$$
\begin{aligned}
& 2(n+1)\left(\frac{p-1}{p}\right)^{1 / p} \int_{q}^{0} \frac{1}{\left(\int_{u}^{q} f(v) d v\right)^{1 / p}} d u \\
& \geq 2(n+1)\left(\frac{p-1}{p}\right)^{1 / p}\left(\frac{p}{f_{0}+\varepsilon}\right)^{1 / p} \\
& \times \int_{q}^{0} \frac{1}{\left((-q)^{p}-(-u)^{p}\right)^{1 / p}} d u \\
& =2(n+1)\left(\frac{p-1}{f_{0}+\varepsilon}\right)^{1 / p} \int_{q}^{0} \frac{1}{\left((-q)^{p}-(-u)^{p}\right)^{1 / p}} d u \\
& =2(n+1)\left(\frac{p-1}{f_{0}+\varepsilon}\right)^{1 / p} \frac{1}{(p-1)^{1 / p}} \\
& \times \int_{0}^{(p-1)^{1 / p}} \frac{1}{\left(1-\left(u^{p} /(p-1)\right)\right)^{1 / p}} d u \\
& =\frac{2(n+1)}{\left(f_{0}+\varepsilon\right)^{1 / p}} \int_{0}^{(p-1)^{1 / p}} \frac{1}{\left(1-\left(u^{p} /(p-1)\right)\right)^{1 / p}} d u \\
& =\frac{(n+1) \pi_{p}}{\left(f_{0}+\varepsilon\right)^{1 / p}} \text {. }
\end{aligned}
$$

Similarly, from the first part of (40), we have that

$$
\begin{aligned}
& 2(n+1)\left(\frac{p-1}{p}\right)^{1 / p} \int_{q}^{0} \frac{1}{\left(\int_{u}^{q} f(v) d v\right)^{1 / p}} d u \\
& \leq 2(n+1)\left(\frac{p-1}{p}\right)^{1 / p}\left(\frac{p}{f_{0}-\varepsilon}\right)^{1 / p} \\
& \times \int_{q}^{0} \frac{1}{\left((-q)^{p}-(-u)^{p}\right)^{1 / p}} d u \\
& =2(n+1)\left(\frac{p-1}{f_{0}-\varepsilon}\right)^{1 / p} \int_{q}^{0} \frac{1}{\left((-q)^{p}-(-u)^{p}\right)^{1 / p}} d u \\
& =2(n+1)\left(\frac{p-1}{f_{0}-\varepsilon}\right)^{1 / p} \frac{1}{(p-1)^{1 / p}} \\
& \times \int_{0}^{(p-1)^{1 / p}} \frac{1}{\left(1-\left(u^{p} /(p-1)\right)\right)^{1 / p}} d u \\
& =\frac{2(n+1)}{\left(f_{0}-\varepsilon\right)^{1 / p}} \int_{0}^{(p-1)^{1 / p}} \frac{1}{\left(1-\left(u^{p} /(p-1)\right)\right)^{1 / p}} d u \\
& =\frac{(n+1) \pi_{p}}{\left(f_{0}-\varepsilon\right)^{1 / p}} \text {. }
\end{aligned}
$$

It follows from (41), (42) and the fact that $\varepsilon$ is arbitrary that we have that

$$
\lim _{q \rightarrow 0} 2\left(\frac{p-1}{p}\right)^{1 / p}(n+1) \int_{q}^{0} \frac{d u}{(F(q)-F(u))^{1 / p}}=\frac{(n+1) \pi_{p}}{f_{0}^{1 / p}} .
$$

Therefore, from (40) and (43), we have that

$$
\lim _{h \rightarrow 0} G_{2 n}(h)=\frac{(2 n+1) \pi_{p}}{f_{0}^{1 / p}} .
$$

By analysing $G 1_{2 n}(h)$ defined in $(20)$ instead of $G_{2 n}(h)$ in the proof of the above, we have the same result. Thus, we have shown that there are two solutions with $2 n$ interior zeros, which are negative near 0 and positive near 0 for $\lambda \in(0,(2 n+$ 1) $\left.{ }^{p} \pi_{p}^{p} / f_{0}\right)$, respectively.

Now, in order to achieve the existence of $\lambda^{*}$, we will first establish that $G_{2 n}^{\prime}(h)<0$ for $h$ large enough. In fact,

$$
\begin{aligned}
G_{2 n}^{\prime}(h)= & 2\left(\frac{p-1}{p}\right)^{1 / p} \\
\times & \left\{n \int_{0}^{1} \frac{H(h)-H(h v)}{(F(h)-F(h v))^{(p+1) / p}} d v\right. \\
& \left.-(n+1) \frac{d q}{d h} \int_{0}^{1} \frac{H(q)-H(q v)}{(F(q)-F(q v))^{(p+1) / p}} d v\right\} .
\end{aligned}
$$

First, we consider

$$
n \int_{0}^{1} \frac{H(h)-H(h v)}{(F(h)-F(h v))^{(p+1) / p}} d v,
$$

where $H(s)=F(s)-(s / p) f(s), H^{\prime}(s)=((p-1) / p) f(s)-$ $(s / p) f^{\prime}(s)$. From the first part of $(\mathrm{H} 2)$, it follows that

$$
H(h)-H(h v) \leq 0, \quad v \in[0,1]
$$

if $h$ is large enough.

Next, let us consider

$$
-(n+1) \frac{d q}{d h} \int_{0}^{1} \frac{H(q)-H(q v)}{(F(q)-F(q v))^{(p+1) / p}} d v,
$$

where $-(d q / d h)>0, H(s)=F(s)-(s / p) f(s), H^{\prime}(s)=((p-$ $1) / p) f(s)-(s / p) f^{\prime}(s)$. From the second part of $(\mathrm{H} 2)$, we have that $H(q)-H(q v) \leq 0$ for $v \in[0,1]$ and $|q|$ large enough. In fact, $q \rightarrow-\infty$ as $h \rightarrow \infty(F(h)=F(q))$. Consequently, we get that $G_{2 n}^{\prime}(h)<0$ for $h$ large enough.

Finally, if $k=2 n$, this clearly follows by analysing $G_{2 n-1}(h)$ defined in (21) instead of $G_{2 n}(h)$ in the proof of the case $k=$ $2 n+1$.

Proof of Theorem 2. First, we consider $k=2 n+1$.

It follows from the quadrature method that a solution with $2 n$ interior zeros exists if for $\lambda>0$ there exists $h \in(0, \infty)$ such that $(\lambda)^{1 / p}=G_{2 n}(h)$. To prove this, we will show that $(0, \infty) \subset \operatorname{Range}\left(G_{2 n}(h)\right)$. We achieve this by proving 

(A1) $\lim _{h \rightarrow+\infty} G_{2 n}(h)=0$
(B1) $\lim _{h \rightarrow 0} G_{2 n}(h)=\infty$.

The proof of (A1) is the same as the proof of (A) of Theorem 1, so we omit it here; we are only to prove (B1). Recall that

$$
\begin{aligned}
G_{2 n}(h)=2\left(\frac{p-1}{p}\right)^{1 / p} & \left\{n \int_{0}^{h} \frac{d u}{(F(h)-F(u))^{1 / p}}\right. \\
& \left.+(n+1) \int_{q}^{0} \frac{d u}{(F(q)-F(u))^{1 / p}}\right\} .
\end{aligned}
$$

First let us consider

$$
\begin{aligned}
& 2\left(\frac{p-1}{p}\right)^{1 / p} n \int_{0}^{h} \frac{d u}{(F(h)-F(u))^{1 / p}} \\
& \quad=2\left(\frac{p-1}{p}\right)^{1 / p} n \int_{0}^{h} \frac{d u}{\left(\int_{u}^{h} f(t) d t\right)^{1 / p}} .
\end{aligned}
$$

From $f_{0}=0$, then, for any $\varepsilon>0$, there exists $\delta \in(0, \infty)$ such that

$$
f(s) \leq \varepsilon^{p} s^{p-1}, \quad 0<s<\delta .
$$

Thus, if $0<h<\delta$, from (50), we have that

$$
\begin{aligned}
2 n & \left(\frac{p-1}{p}\right)^{1 / p} \int_{0}^{h} \frac{1}{\left(\int_{u}^{h} f(v) d v\right)^{1 / p}} d u \\
\geq & 2 n\left(\frac{p-1}{p}\right)^{1 / p} \frac{p^{1 / p}}{\varepsilon} \int_{0}^{h} \frac{1}{\left(h^{p}-u^{p}\right)^{1 / p}} d u \\
= & 2 n \frac{(p-1)^{1 / p}}{\varepsilon} \int_{0}^{1} \frac{1}{\left(1-(u / h)^{p}\right)^{1 / p}} d\left(\frac{u}{h}\right) \\
= & 2 n \frac{(p-1)^{1 / p}}{\varepsilon} \frac{1}{(p-1)^{1 / p}} \\
& \times \int_{0}^{(p-1)^{1 / p}} \frac{1}{\left(1-\left(s^{p} /(p-1)\right)\right)^{1 / p}} d s \\
= & \frac{2 n}{\varepsilon} \int_{0}^{(p-1)^{1 / p}} \frac{1}{\left(1-\left(s^{p} /(p-1)\right)\right)^{1 / p}} d s \\
= & \frac{n}{\varepsilon} \pi_{p} .
\end{aligned}
$$

Next, in fact, $q \rightarrow 0$ as $h \rightarrow 0(F(q)=F(h))$; we consider

$$
2\left(\frac{p-1}{p}\right)^{1 / p}(n+1) \int_{q}^{0} \frac{d u}{(F(q)-F(u))^{1 / p}} .
$$

Since $f_{0}=0$, then, for any $\varepsilon>0$, there exists $\delta \in(0, \infty)$ such that

$$
-f(s) \leq \varepsilon^{p}|s|^{p-1}, \quad-\delta<s<0 .
$$

Thus, if $-\delta<q<0$, from (54), we have that

$$
\begin{aligned}
2\left(\frac{p-1}{p}\right)^{1 / p}(n+1) \int_{q}^{0} \frac{d u}{\left(\int_{u}^{q} f(t) d t\right)^{1 / p}} \\
\geq 2(n+1)\left(\frac{p-1}{p}\right)^{1 / p} \frac{p^{1 / p}}{\varepsilon} \\
\quad \times \int_{q}^{0} \frac{1}{\left((-q)^{p}-(-u)^{p}\right)^{1 / p}} d u \\
=2(n+1) \frac{(p-1)^{1 / p}}{\varepsilon} \int_{0}^{1} \frac{1}{\left(1-(u / q)^{p}\right)^{1 / p}} d\left(\frac{u}{q}\right) \\
=2(n+1) \frac{(p-1)^{1 / p}}{\varepsilon} \frac{1}{(p-1)^{1 / p}} \\
\quad \times \int_{0}^{(p-1)^{1 / p}} \frac{1}{\left(1-\left(v^{p} /(p-1)\right)\right)^{1 / p}} d v \\
=\frac{2(n+1)}{\varepsilon} \int_{0}^{(p-1)^{1 / p}} \frac{1}{\left(1-\left(v^{p} /(p-1)\right)\right)^{1 / p}} d v \\
=\frac{n+1}{\varepsilon} \pi_{p} .
\end{aligned}
$$

From the fact that $\varepsilon$ is small and combining (52) and (55), we get that

$$
\lim _{h \rightarrow 0} G_{2 n}(h)=\infty .
$$

By analyzing $G 1_{2 n}(h)$ defined in $(20)$ instead of $G_{2 n}(h)$ in the proof of the above, we have the same result. The proof of $\lambda^{*}$ is similar to the proof of Theorem 1 . We omit it here.

Finally, if $k=2 n$, then it clearly follows by analyzing $G_{2 n-1}(h)$ defined in (21) instead of $G_{2 n}(h)$ in the proof of the case $k=2 n+1$.

Proof of Theorem 3. First, we consider $k=2 n+1$.

It follows from the quadrature method that a solution with $2 n$ interior zeros exists if for $\lambda>0$ there exists $h \in(0, \infty)$ such that $(\lambda)^{1 / p}=G_{2 n}(h)$. We prove this by proving

$$
\begin{aligned}
& \text { (A2) } \lim _{h \rightarrow \infty} G_{2 n}(h)=0, \\
& \text { (B2) } \lim _{h \rightarrow 0} G_{2 n}(h)=0 .
\end{aligned}
$$

The proof of (A2) is the same as the proof of (A) of Theorem 1, so we omit it here; we are only to prove (B2).

Recall that

$$
\begin{aligned}
G_{2 n}(h)=2\left(\frac{p-1}{p}\right)^{1 / p} & \left\{n \int_{0}^{h} \frac{d u}{(F(h)-F(u))^{1 / p}}\right. \\
& \left.+(n+1) \int_{q}^{0} \frac{d u}{(F(q)-F(u))^{1 / p}}\right\} .
\end{aligned}
$$


First, consider

$$
\begin{aligned}
& 2\left(\frac{p-1}{p}\right)^{1 / p} n \int_{0}^{h} \frac{d u}{(F(h)-F(u))^{1 / p}} \\
& \quad=2\left(\frac{p-1}{p}\right)^{1 / p} n \int_{0}^{h} \frac{d u}{\left(\int_{u}^{h} f(t) d t\right)^{1 / p}} .
\end{aligned}
$$

Since $f_{0}=\infty$, then, for any large $\epsilon$, there exists $R \in(0, \infty)$ such that

$$
f(s) \geq \epsilon^{p} s^{p-1}, \quad 0<s<R .
$$

Thus, if $0<h<R$, from (58) and (59), we have that

$$
\begin{aligned}
& 2\left(\frac{p-1}{p}\right)^{1 / p} n \int_{0}^{h} \frac{d u}{\left(\int_{u}^{h} f(t) d t\right)^{1 / p}} \\
& =2 n\left(\frac{p-1}{p}\right)^{1 / p} \int_{0}^{h} \frac{1}{\left(\int_{u}^{h} f(t) d t\right)^{1 / p}} d u \\
& \leq 2 n\left(\frac{p-1}{p}\right)^{1 / p} \frac{p^{1 / p}}{\epsilon} \int_{0}^{h} \frac{1}{\left(h^{p}-u^{p}\right)^{1 / p}} d u \\
& =2 n \frac{(p-1)^{1 / p}}{\epsilon} \int_{0}^{1} \frac{1}{\left(1-(u / h)^{p}\right)^{1 / p}} d\left(\frac{u}{h}\right) \\
& =2 n \frac{(p-1)^{1 / p}}{\epsilon} \frac{1}{(p-1)^{1 / p}} \\
& \times \int_{0}^{(p-1)^{1 / p}} \frac{1}{\left(1-\left(s^{p} /(p-1)\right)\right)^{1 / p}} d s \\
& =\frac{2 n}{\epsilon} \int_{0}^{(p-1)^{1 / p}} \frac{1}{\left(1-\left(s^{p} /(p-1)\right)\right)^{1 / p}} d s \\
& =\frac{n}{\epsilon} \pi_{p} .
\end{aligned}
$$

Next, in fact, $q \rightarrow 0$ as $h \rightarrow 0(F(q)=F(h))$; we consider

$$
2\left(\frac{p-1}{p}\right)^{1 / p}(n+1) \int_{q}^{0} \frac{d u}{(F(q)-F(u))^{1 / p}} .
$$

Since $f_{0}=\infty$, then, for any large $\epsilon$, there exists $R \in(0, \infty)$ such that

$$
-f(s) \geq \epsilon^{p}|s|^{p-1}, \quad-R<s<0 .
$$

Thus, if $-R<q<0$, from (62), we have that

$$
\begin{aligned}
& 2(n+1)\left(\frac{p-1}{p}\right)^{1 / p} \int_{q}^{0} \frac{1}{\left(\int_{u}^{q} f(t) d t\right)^{1 / p}} d u \\
& \quad \leq 2(n+1)\left(\frac{p-1}{p}\right)^{1 / p} \frac{p^{1 / p}}{\epsilon} \int_{q}^{0} \frac{1}{\left((-q)^{p}-(-u)^{p}\right)^{1 / p}} d u
\end{aligned}
$$

$$
\begin{aligned}
& =2(n+1) \frac{(p-1)^{1 / p}}{\epsilon} \int_{0}^{1} \frac{1}{\left(1-(u / q)^{p}\right)^{1 / p}} d\left(\frac{u}{q}\right) \\
& =\frac{2(n+1)}{\epsilon} \int_{0}^{(p-1)^{1 / p}} \frac{1}{\left(1-\left(s^{p} /(p-1)\right)\right)^{1 / p}} d s=\frac{n+1}{\epsilon} \pi_{p} .
\end{aligned}
$$

From the fact that $\epsilon$ is arbitrary and large and combining (60) and (63), we get that

$$
\lim _{h \rightarrow 0} G_{2 n}(h)=0
$$

By analyzing $G 1_{2 n}(h)$ defined in $(20)$ instead of $G_{2 n}(h)$ in the proof of the above, we have the same result.

Finally, if $k=2 n$, then it clearly follows by analyzing $G_{2 n-1}(h)$ defined in (20) instead of $G_{2 n}(h)$ in the proof of the case $k=2 n+1$.

\section{Conflict of Interests}

The authors declare that there is no conflict of interests regarding the publication of this paper.

\section{Acknowledgments}

This work is supported by the NSFC (no. 11361054), SRFDP (no. 20126203110004), and Gansu Provincial National Science Foundation of China (no. 1208RJZA258).

\section{References}

[1] L. H. Erbe and H. Wang, "On the existence of positive solutions of ordinary differential equations," Proceedings of the American Mathematical Society, vol. 120, no. 3, pp. 743-748, 1994.

[2] J. Henderson and H. Wang, "Positive solutions for nonlinear eigenvalue problems," Journal of Mathematical Analysis and Applications, vol. 208, no. 1, pp. 252-259, 1997.

[3] T. Laetsch, "The number of solutions of a nonlinear two point boundary value problem," Indiana University Mathematics Journal, vol. 20, pp. 1-13, 1971.

[4] A. M. Fink, J. A. Gatica, and G. E. Hernández, "Eigenvalues of generalized Gel'fand models," Nonlinear Analysis: Theory, Methods \& Applications, vol. 20, no. 12, pp. 1453-1468, 1993.

[5] R. Ma and B. Thompson, "Multiplicity results for secondorder two-point boundary value problems with superlinear or sublinear nonlinearities," Journal of Mathematical Analysis and Applications, vol. 303, no. 2, pp. 726-735, 2005.

[6] R. Ma, "Nodal solutions of second-order boundary value problems with superlinear or sublinear nonlinearities," Nonlinear Analysis: Theory, Methods \& Applications, vol. 66, no. 4, pp. 950961, 2007

[7] Y. Lee and I. Sim, "Global bifurcation phenomena for singular one-dimensional p-Laplacian," Journal of Differential Equations, vol. 229, no. 1, pp. 229-256, 2006.

[8] J. Wang, "The existence of positive solutions for the onedimensional $p$-Laplacian," Proceedings of the American Mathematical Society, vol. 125, no. 8, pp. 2275-2283, 1997. 
[9] L. Kong and J. Wang, "Multiple positive solutions for the onedimensional $p$-Laplacian," Nonlinear Analysis: Theory, Methods \& Applications, vol. 42, no. 8, pp. 1327-1333, 2000.

[10] C. Aranda and T. Godoy, "Existence and multiplicity of positive solutions for a singular problem associated to the $p$-Laplacian operator," Electronic Journal of Differential Equations, vol. 2004, no. 132, 15 pages, 2004.

[11] S. M. Bouguima and A. Lakmeche, "Multiple solutions of a nonlinear problem involving the $p$-Laplacian," Communications on Applied Nonlinear Analysis, vol. 7, no. 3, pp. 83-96, 2000.

[12] C. de Coster, "Pairs of positive solutions for the onedimensional p-Laplacian," Nonlinear Analysis, vol. 23, no. 5, pp. 669-681, 1994.

[13] Y. Lee and I. Sim, "Existence results of sign-changing solutions for singular one-dimensional p-Laplacian problems," Nonlinear Analysis: Theory, Methods \& Applications, vol. 68, no. 5, pp. 11951209, 2008.

[14] W. Reichel and W. Walter, "Radial solutions of equations and inequalities involving the $p$-Laplacian," Journal of Inequalities and Applications, vol. 1, no. 1, pp. 47-71, 1997.

[15] V. Anuradha and R. Shivaji, "Existence of infinitely many nontrivial bifurcation points," Results in Mathematics, vol. 22, no. 3-4, pp. 641-650, 1992.

[16] L. Gasińki and N. S. Papageorgiou, "Bifurcation-type results for nonlinear parametric elliptic equations," Proceedings of the Royal Society of Edinburgh A: Mathematics, vol. 142, no. 3, pp. 595-623, 2012.

[17] L. Gasiński, "Positive solutions for resonant boundary value problems with the scalar $p$-Laplacian and nonsmooth potential," Discrete and Continuous Dynamical Systems A, vol. 17, no. 1, pp. 143-158, 2007.

[18] M. Filippakis, L. Gasiski, and N. S. Papageorgiou, "Multiple positive solutions for eigenvalue problems of hemivariational inequalities," Positivity, vol. 10, no. 3, pp. 491-515, 2006.

[19] A. Castro and R. Shivaji, "Nonnegative solutions for a class of nonpositone problems," Proceedings of the Royal Society of Edinburgh A, vol. 108, no. 3-4, pp. 291-302, 1988.

[20] Z. Liu and F. Li, "Multiple positive solutions of nonlinear two-point boundary value problems," Journal of Mathematical Analysis and Applications, vol. 203, no. 3, pp. 610-625, 1996.

[21] P. Drabek, P. Girg, P. Takac, and M. Ulm, “The Fredholm alternative for the $p$-Laplacian: bifurcation from infinity, existence and multiplicity," Indiana University Mathematics Journal, vol. 53, no. 2, pp. 433-482, 2004.

[22] K. J. Brown, M. M. A. Ibrahim, and R. Shivaji, "S-shaped bifurcation curves," Nonlinear Analysis, vol. 5, no. 5, pp. 475486, 1981.

[23] R. Manásevich and F. Zanolin, "Time-mappings and multiplicity of solutions for the one-dimensional $p$-Laplacian," Nonlinear Analysis: Theory, Methods \& Applications, vol. 21, no. 4, pp. 269291, 1993.

[24] I. Addou, S. M. Bouguima, M. Derhab, and Y. Raffed, "Multiple solutions of Dirichlet problems," Dynamic Systems an Applications, vol. 7, no. 4, pp. 575-601, 1998. 


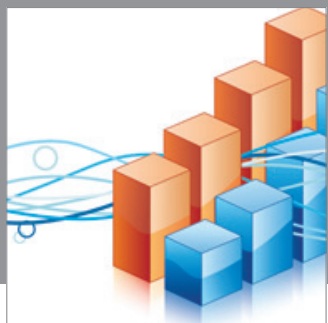

Advances in

Operations Research

mansans

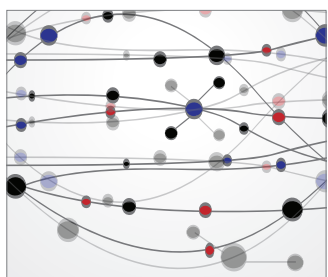

The Scientific World Journal
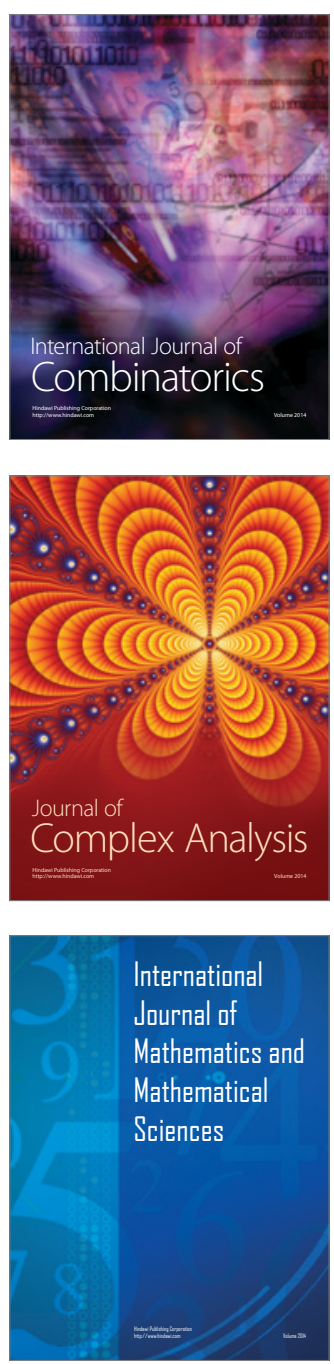
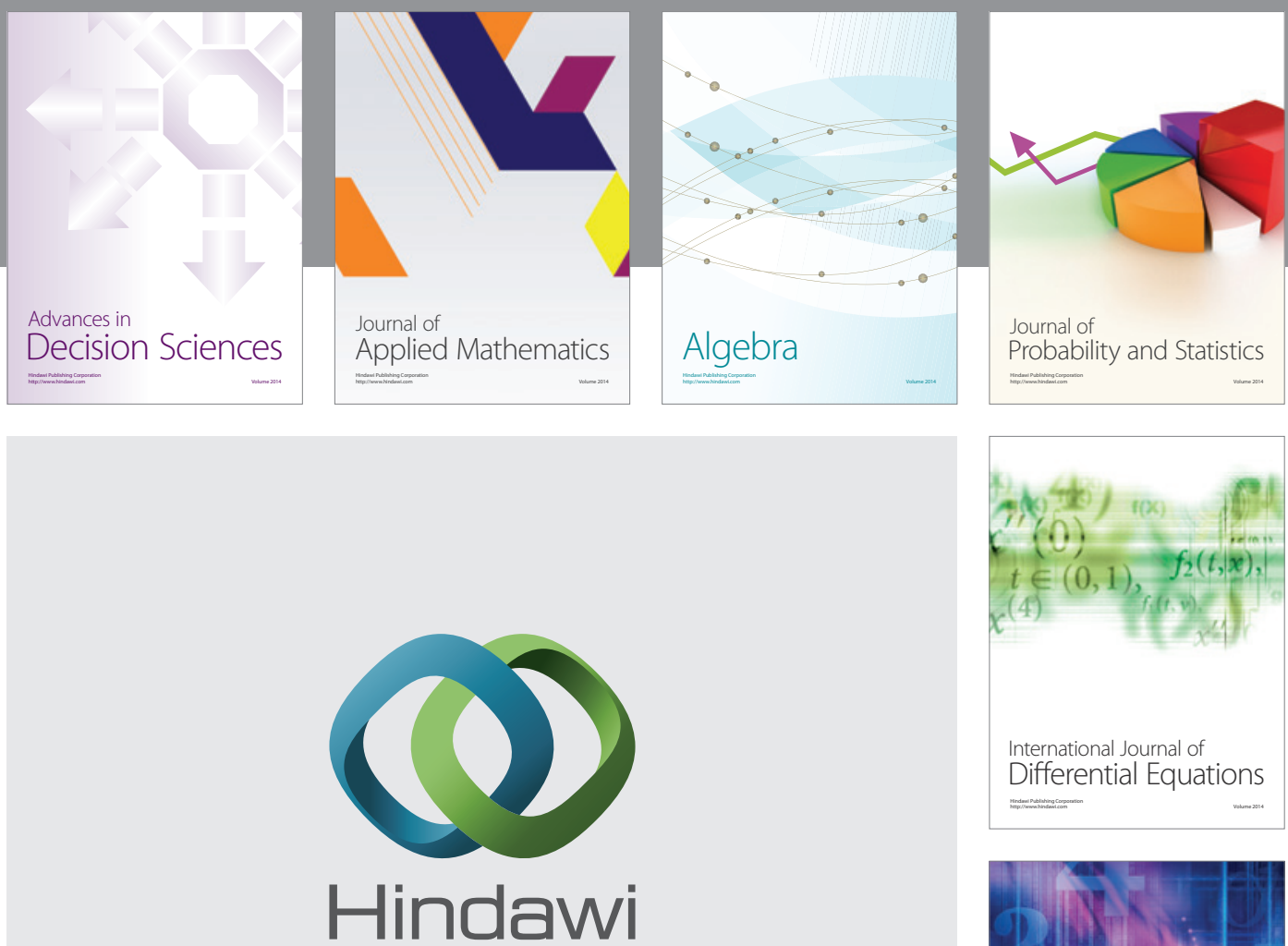

Submit your manuscripts at http://www.hindawi.com
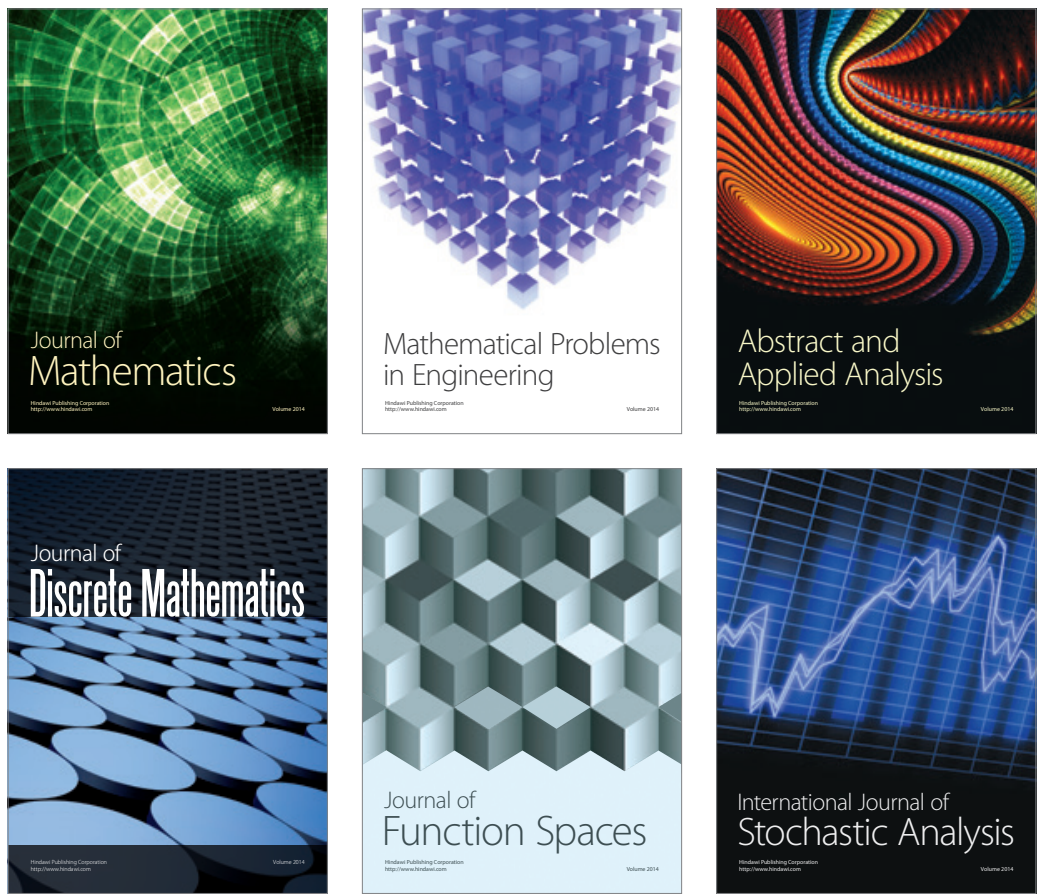

Journal of

Function Spaces

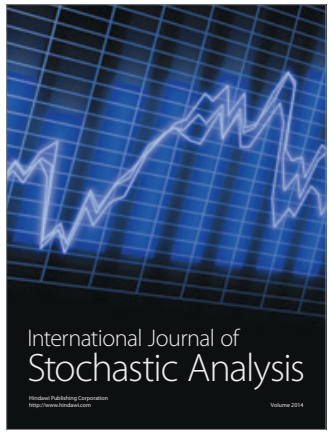

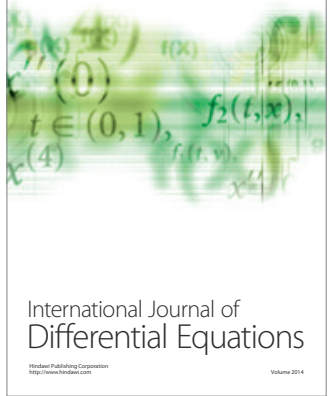
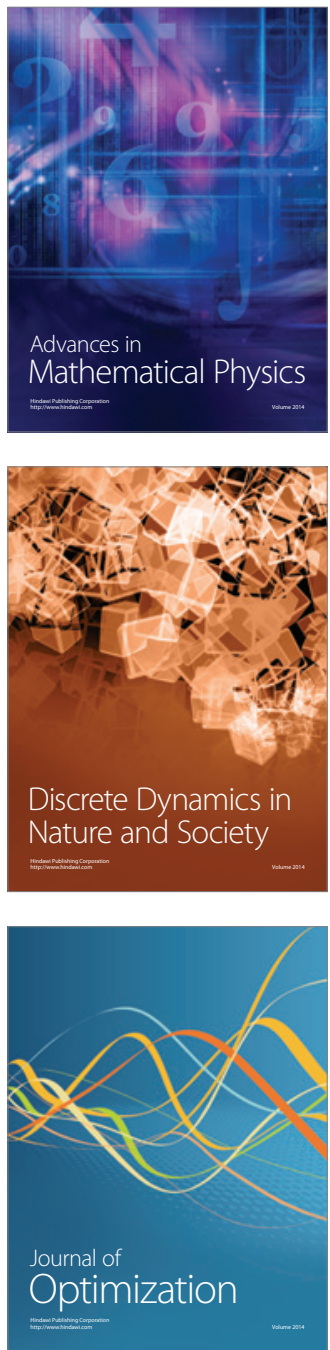